\title{
Chemotherapy treatment patterns and neutropenia management in gastric cancer
}

\author{
Ewa Kalinka-Warzocha • Javier Gallego Plazas • \\ Laurent Mineur · Tomas Salek • Alain Hendlisz • \\ Lucy DeCosta $\cdot$ Florian D. Vogl $\cdot$ Rodolfo Passalacqua
}

Received: 4 December 2013/ Accepted: 28 March 2014/Published online: 5 May 2014

(c) The International Gastric Cancer Association and The Japanese Gastric Cancer Association 2014

\begin{abstract}
Background Potentially myelosuppressive doublet and triplet chemotherapy combination regimens are considered the most active treatments in gastric cancer. This multicenter prospective observational study was designed to gain insight into the chemotherapy regimens being used in Europe and to evaluate neutropenia management in patients identified as at high risk for febrile neutropenia (FN).

Methods Eligible patients had gastric cancer, were scheduled for $\geq 3$ cycles of myelosuppressive chemotherapy, and had an investigator-assessed overall FN risk $\geq 20 \%$. Data were collected for up to ten cycles. The primary endpoint was the proportion of patients who received granulocyte colony
\end{abstract}

E. Kalinka-Warzocha $(\bowtie)$

Wojewodzki Szpital, Pabianicka 62, 93-513 Lodz, Poland

e-mail: ewakalinka@wp.pl

J. G. Plazas

Hospital General Universitario de Elche, Elche, Spain

L. Mineur

Clinique Sainte Catherine, Sainte Catherine Institut, Avignon,

France

T. Salek

Narodny Onkologicky Ustav, Bratislava, Slovakia

A. Hendlisz

Institut Jules Bordet, Brussels, Belgium

L. DeCosta

Amgen, Uxbridge, UK

F. D. Vogl

Amgen Europe GmbH, Zug, Switzerland

R. Passalacqua

Istituti Ospitalieri di Cremona, Cremona, Italy stimulating factor (G-CSF) primary prophylaxis (defined as G-CSF initiated on days 1-7 of cycle 1). Secondary endpoints included FN incidence, chemotherapy administration, and G-CSF use.

Results Of 199 patients who met the eligibility criteria and started at least one cycle of chemotherapy, mean age was 63 years, $76 \%$ were men, $83 \%$ had an ECOG score of 0 or 1 , $54 \%$ had metastatic disease, and $24 \%$ had received prior chemotherapy. A total of 27 different backbone regimens were given; the most common regimen was modified docetaxel, cisplatin, and 5-fluorouracil (DCF). Despite all patients having been identified as having $\mathrm{a} \geq 20 \%$ FN risk, only 70 $(35 \%)$ received G-CSF primary prophylaxis. FN occurred in 14 patients overall ( $7 \%$ ). Most FN events occurred in patients who received DCF/modified DCF (9/14 events, $64 \%$ ).

Conclusions The results of this study reveal a high use of myelotoxic treatment regimens in gastric cancer in Europe and low adherence to clinical practice guidelines for the use of primary and secondary G-CSF prophylaxis for FN.

Keywords Febrile neutropenia - Gastric cancer . Granulocyte colony stimulating factor - Myelosuppressive chemotherapy $\cdot$ Primary prophylaxis · Observational research

\section{Introduction}

Despite a marked decline in the incidence of gastric cancer in recent decades, it remains a leading cause of death, as most cases are diagnosed at an advanced stage and survival is poor. Worldwide, the highest incidences of gastric cancer are found in Eastern Asia, Central and Eastern Europe, and South America [1]. In Europe, the estimated 2012 incidence of gastric cancer is 139,595 cases, with 107,268 
projected deaths [2]. The standard treatment approach in patients with localized disease is surgical resection with perioperative chemotherapy, while palliative chemotherapy is used in patients with locally advanced inoperable disease and in those with metastatic disease [3-5]. No single chemotherapy regimen has yet emerged as a global standard in gastric cancer. At the present time, double and triple combinations including 5-fluorouracil (5-FU), cisplatin \pm anthracyclines as well as docetaxel-containing or irinotecan-containing regimens are considered the most active treatments [3, 4, 6, 7]. In patients with HER-2 overexpression, the addition of trastuzumab to cisplatin/ fluoropyrimidine has been shown to be superior to chemotherapy alone [8]. In patients over 65, fluorouracil, leucovorin, and oxaliplatin improve toxicity and efficacy compared with fluorouracil, leucovorin, and cisplatin [9].

While combination regimens are more effective than single agents, they are associated with increased toxicity. In particular, chemotherapy combinations may be considerably more myelosuppressive; for example, DCF (docetaxel, cisplatin, and 5-FU) is associated with $\mathrm{FN}$ and/or neutropenic infection in $29 \%$ of patients [10]. FN can be life-threatening, with up to $9.5 \%$ in-hospital mortality reported [11]. FN can lead to reduced chemotherapy dose delivery and subsequently, reduced chemotherapy efficacy [12-14]. Guidelines recommend use of G-CSF primary prophylaxis in patients with $\geq 20 \%$ risk of FN based on chemotherapy regimen and individual patient characteristics, while secondary prophylaxis is recommended in the subsequent cycle after an FN event $[15,16]$. At the time of initiation of the present study in 2009, the European Organisation for Research and Treatment of Cancer (EORTC) guidelines for the use of G-CSF to reduce the incidence of chemotherapy-induced FN did not list any regimens for gastric cancer [16]. However, in the 2010 updated guidelines, a few regimens for use in metastatic gastric cancer were listed, and these included DCF, TCF (docetaxel, cisplatin, 5-FU), and TC (docetaxel, cisplatin), which were assigned to the high FN risk category [15].

The risk of FN associated with gastric cancer regimens has not been well studied and under-reporting of $\mathrm{FN}$ and G-CSF use in clinical studies is common [17]; therefore, we undertook an observational study to gain insight into the types of chemotherapy regimens being used in gastric cancer in Europe, and to evaluate neutropenia management in patients identified by the investigator as at high risk for FN.

\section{Methods}

Study design

IMPACT Gastric was a multicenter prospective observational study conducted from November 2009 to
January 2012. Patients were enrolled from 29 centers in seven countries including Belgium, Czech Republic, France, Italy, Poland, Slovakia, and Spain. Investigational sites were not required to use G-CSFs to participate. Patients had to provide appropriate written informed consent and the protocol was approved by ethical committees and regulatory authorities as required by the country. Data were collected for up to a maximum of ten chemotherapy cycles.

\section{Patients}

Adult patients with gastric cancer (any stage) who were scheduled to receive $\geq 3$ consecutive cycles of myelosuppressive chemotherapy and had an investigator-assessed overall FN risk $\geq 20 \%$ (based on the EORTC 2006 guidelines [16] current at that time) were eligible. Patients were excluded if they were scheduled for concurrent administration of radiotherapy or for a gastric cancer chemotherapy regimen for which use of G-CSF was contraindicated, or if they were enrolled in another clinical study in which G-CSF use was specified in the protocol.

\section{Outcome measures}

The primary endpoint was the proportion of patients who received G-CSF primary prophylaxis (defined as G-CSF initiated on days 1-7 of cycle 1). Secondary endpoints included FN incidence, chemotherapy administration, G-CSF use and safety. Post hoc analyses investigated the subgroup of patients who received DCF, including modifications from standard DCF, and the extent of G-CSF support given up to the first episode of FN. An FN event was defined as a single oral temperature of $\geq 38.3{ }^{\circ} \mathrm{C}$ or a temperature of $\geq 38.0{ }^{\circ} \mathrm{C}$ for $\geq 1 \mathrm{~h}$, with a neutrophil count of $0.5 \times 10^{9} / \mathrm{L}$ or lower, or neutrophil count $>0.5 \times 10^{9} / \mathrm{L}$ and $\leq 1.0 \times 10^{9} / \mathrm{L}$ and expected to fall below $0.5 \times 10^{9} / \mathrm{L}$.

\section{Statistical analyses}

The prespecified analysis of overall G-CSF use was derived programmatically, and each patient was included in one of the following groups:

- G-CSF primary prophylaxis, defined as G-CSF administration initiating on days 1 to 7 of cycle 1 ;

- G-CSF secondary prophylaxis, defined as initiating administration on days 1 to 7 of cycle 2 or later;

- G-CSF treatment, defined as initiation of administration after day 7 of any cycle;

- No G-CSF use, defined as receiving no G-CSF, either as prophylaxis or treatment. 
The No G-CSF primary prophylaxis group was defined as all patients not contained within the $G-C S F$ primary prophylaxis group.

Post hoc analyses were conducted to examine the extent of prophylactic G-CSF use at the time of an FN event. These analyses applied a narrower definition of prophylaxis and required G-CSF prophylaxis in all cycles after first administration, and were more closely aligned to the pegfilgrastim (Neulasta ${ }^{\circledR}$ ) and filgrastim (Neupogen ${ }^{\circledR}$ ) Summary of Product Characteristics [18, 19]. In this instance, prophylactic G-CSF was defined as G-CSF initiated 1 to 3 days after the end of chemotherapy for regimens not containing 5-FU, and G-CSF initiated 1 to 3 days after the end of 5-FU administration for regimens containing 5-FU. For the purposes of this report, we have termed this definition "on-schedule" prophylaxis to distinguish it from the prespecified analyses. For daily G-CSF, $\geq 3$ days of continuous administration were required to be considered onschedule prophylaxis use. Each patient who experienced an FN event was included in one of the following groups:

- On-schedule primary prophylaxis, defined as prophylactic G-CSF from cycle 1 and in every subsequent cycle until the FN event occurred;

- On-schedule secondary prophylaxis, defined as prophylactic G-CSF started in cycle 2 or later and given in every subsequent cycle until the FN event occurred;

- No on-schedule prophylaxis, defined as no prophylactic G-CSF given in the cycle in which the FN event occurred.

In addition, we evaluated whether patients who had experienced FN received on-schedule G-CSF prophylaxis in the next cycle. Other post hoc analyses were conducted to characterize the G-CSF use and FN incidence in patients receiving either standard DCF regimens (defined as docetaxel $75 \mathrm{mg} /$ $\mathrm{m}^{2}$, cisplatin $75 \mathrm{mg} / \mathrm{m}^{2}$, and a total dose of $3750 \mathrm{mg} / \mathrm{m}^{2}$ of 5 -FU in cycle 1) [10], allowing up to a $10 \%$ dose reduction in any agent, or modified (non-standard) DCF regimens. G-CSF use in the study was with either once-per-cycle pegfilgrastim or with a daily G-CSF; patients were allowed to receive any daily G-CSF (filgrastim, lenograstim, or filgrastim biosimilars) and results for daily G-CSF use were grouped together. The intent of chemotherapy was determined based on TNM staging collected on the CRF as follows: palliative (M+), nonpalliative (M0, T2 or higher, any N), and other. All statistical analyses were descriptive in nature.

\section{Results}

Patients and disposition

Of 209 patients enrolled, 199 (95\%) met the eligibility criteria and started at least one cycle of chemotherapy and were included in the full analysis set (FAS). The most common reason for exclusion from the FAS was failure to start at least one cycle of chemotherapy ( $n=5$; Fig. 1 ). Thirty-six percent of patients $(n=75)$ discontinued the study prematurely, with the most common reasons being death $(25 \%)$, disease progression $(20 \%)$, non-hematologic adverse event $(17 \%)$, and other $(19 \%)$. The mean age was 63 years and most $(76 \%)$ patients were men (Table 1). Most patients (83\%) had an ECOG score of
Fig. 1 CONSORT diagram. ${ }^{a}$ Requiring or leading to discontinuation of chemotherapy

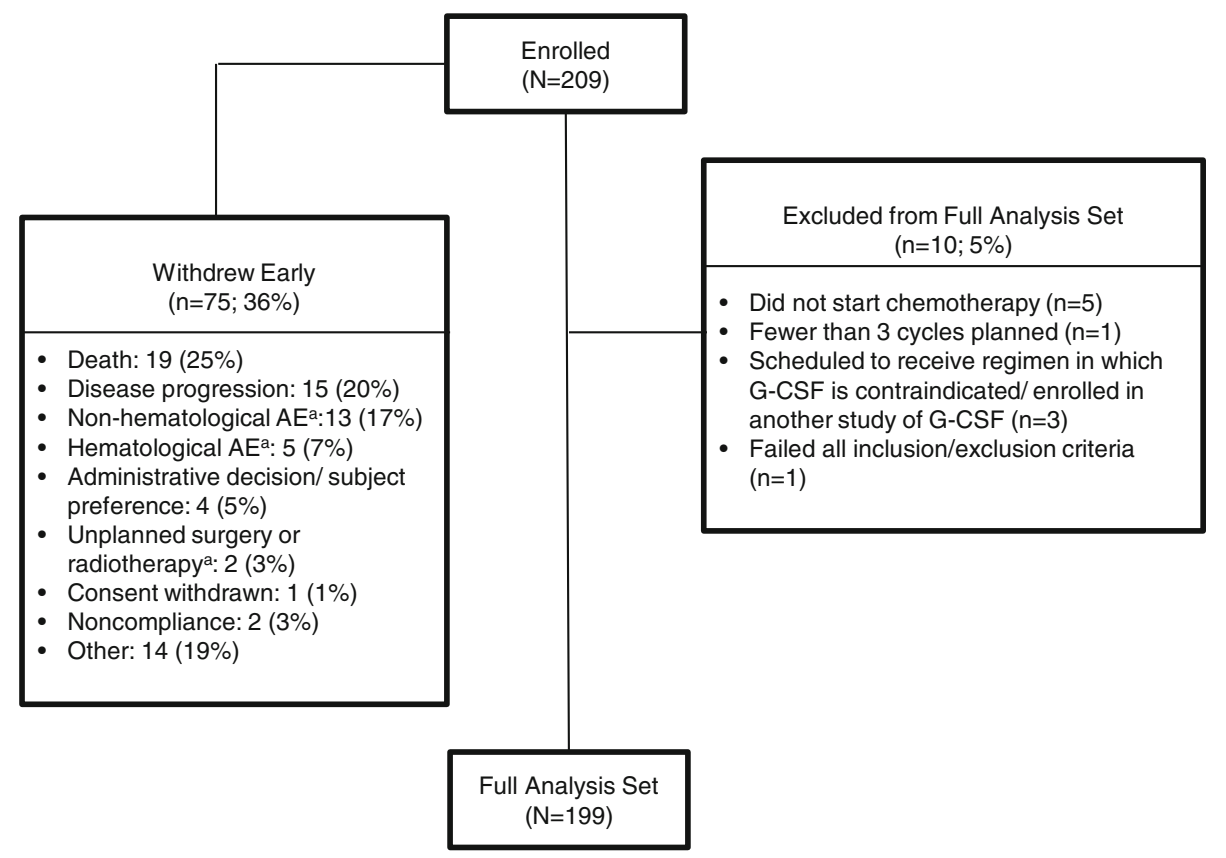


Table 1 Baseline demographics and disease characteristics

\begin{tabular}{|c|c|c|c|}
\hline & $\begin{array}{l}\text { Received G-CSF primary } \\
\text { prophylaxis }(N=70)\end{array}$ & $\begin{array}{l}\text { No G-CSF primary } \\
\text { prophylaxis }(N=129)\end{array}$ & $\begin{array}{l}\text { Total } \\
(N=199)\end{array}$ \\
\hline \multicolumn{4}{|l|}{ Age, years } \\
\hline Mean (SD) & $60(14)$ & $64(10)$ & $63(12)$ \\
\hline Male, $n(\%)$ & $54(77 \%)$ & $98(76 \%)$ & $152(76 \%)$ \\
\hline \multicolumn{4}{|l|}{ ECOG score at baseline, $n(\%)$} \\
\hline $0-1$ & $60(86 \%)$ & $105(81 \%)$ & $165(83 \%)$ \\
\hline $2-3$ & $9(13 \%)$ & $24(19 \%)$ & $33(17 \%)$ \\
\hline 4 & 0 & 0 & 0 \\
\hline Missing & $1(1 \%)$ & 0 & $1(1 \%)$ \\
\hline \multicolumn{4}{|l|}{ AJCC staging at baseline, $n(\%)$} \\
\hline Stage 0 & 0 & 0 & 0 \\
\hline Stage I & $1(1 \%)$ & $3(2 \%)$ & $4(2 \%)$ \\
\hline Stage II & $5(7 \%)$ & $11(9 \%)$ & $16(8 \%)$ \\
\hline Stage III & $13(19 \%)$ & $22(17 \%)$ & $35(18 \%)$ \\
\hline Stage IV & $45(64 \%)$ & $81(63 \%)$ & $126(63 \%)$ \\
\hline Other $^{\mathrm{a}}$ & $5(7 \%)$ & $9(7 \%)$ & $14(7 \%)$ \\
\hline Missing & $1(1 \%)$ & $3(2 \%)$ & $4(2 \%)$ \\
\hline \multicolumn{4}{|l|}{ TNM disease staging } \\
\hline \multicolumn{4}{|l|}{ Primary tumor $(\mathrm{T}), n(\%)$} \\
\hline $\mathrm{TX}$ & $12(17 \%)$ & $33(26 \%)$ & $45(23 \%)$ \\
\hline $\mathrm{T} 1$ & $3(4 \%)$ & $0(0 \%)$ & $3(2 \%)$ \\
\hline $\mathrm{T} 2$ & $9(13 \%)$ & $16(12 \%)$ & $25(13 \%)$ \\
\hline $\mathrm{T} 3$ & $24(34 \%)$ & $54(42 \%)$ & $78(39 \%)$ \\
\hline $\mathrm{T} 4$ & $21(30 \%)$ & $23(18 \%)$ & $44(22 \%)$ \\
\hline Missing & $1(1 \%)$ & $3(2 \%)$ & $4(2 \%)$ \\
\hline \multicolumn{4}{|l|}{ Regional lymph nodes $(\mathrm{N}), n(\%)$} \\
\hline NX & $18(26 \%)$ & $40(31 \%)$ & $58(29 \%)$ \\
\hline No & $4(6 \%)$ & $10(8 \%)$ & $14(7 \%)$ \\
\hline N1 & $21(30 \%)$ & $37(29 \%)$ & $58(29 \%)$ \\
\hline $\mathrm{N} 2$ & $14(20 \%)$ & $20(16 \%)$ & $34(17 \%)$ \\
\hline $\mathrm{N} 3$ & $12(17 \%)$ & $19(15 \%)$ & $31(16 \%)$ \\
\hline Missing & $1(1 \%)$ & $3(2 \%)$ & $4(2 \%)$ \\
\hline \multicolumn{4}{|l|}{ Distant metastases (M), $n(\%)$} \\
\hline MX & $9(13 \%)$ & $8(6 \%)$ & $17(9 \%)$ \\
\hline M0 & $24(34 \%)$ & $46(36 \%)$ & $70(35 \%)$ \\
\hline M1 & $36(51 \%)$ & $72(56 \%)$ & $108(54 \%)$ \\
\hline Missing & $1(1 \%)$ & $3(2 \%)$ & $4(2 \%)$ \\
\hline \multicolumn{4}{|l|}{ Prior gastric tumor treatment, $n(\%)$} \\
\hline Naïve & $29(41 \%)$ & $65(50 \%)$ & $94(47 \%)$ \\
\hline Previously treated & $41(59 \%)$ & $64(50 \%)$ & $105(53 \%)$ \\
\hline Chemotherapy \pm radiotherapy & $11 / 41(27 \%)$ & $14 / 64(22 \%)$ & $25 / 105(24 \%)$ \\
\hline Surgery & $23 / 41(56 \%)$ & $40 / 64(63 \%)$ & $63 / 105(60 \%)$ \\
\hline \multicolumn{4}{|c|}{ Type of planned chemotherapy ${ }^{\mathrm{b}}, n(\%)$} \\
\hline Palliative & $36(51 \%)$ & $72(56 \%)$ & $108(54 \%)$ \\
\hline Non-palliative & $23(33 \%)$ & $44(34 \%)$ & $67(34 \%)$ \\
\hline Other & $11(16 \%)$ & $13(10 \%)$ & $24(12 \%)$ \\
\hline \multicolumn{4}{|l|}{ Primary tumor location, $n(\%)$} \\
\hline Gastric & $59(84 \%)$ & $106(82 \%)$ & $165(83 \%)$ \\
\hline Distal esophageal & $3(4 \%)$ & $8(6 \%)$ & $11(6 \%)$ \\
\hline
\end{tabular}


Table 1 continued

\begin{tabular}{llll}
\hline & $\begin{array}{l}\text { Received G-CSF primary } \\
\text { prophylaxis }(N=70)\end{array}$ & $\begin{array}{l}\text { No G-CSF primary } \\
\text { prophylaxis }(N=129)\end{array}$ & $\begin{array}{l}\text { Total } \\
(N=199)\end{array}$ \\
\hline Gastroesophageal junction & $8(11 \%)$ & $15(12 \%)$ & $23(12 \%)$ \\
Comorbidities ${ }^{c}, n$ & 32 & 55 & 87 \\
Cardiovascular, $n(\%)$ & $25(78 \%)$ & $35(64 \%)$ & $60(69 \%)$ \\
Endocrine/metabolic, $n(\%)$ & $5(16 \%)$ & $21(38 \%)$ & $26(30 \%)$ \\
Gastrointestinal, $n(\%)$ & $4(13 \%)$ & $7(13 \%)$ & $3(13 \%)$ \\
Hepatic/biliary, $n(\%)$ & $1(3 \%)$ & $2(4 \%)$ & $3(3 \%)$ \\
Renal, $n(\%)$ & 0 & $3(5 \%)$ & $3(3 \%)$ \\
\hline
\end{tabular}

${ }^{a}$ Other includes: TX-NX-M0/MX, TX-N1/N3-M0, T3/T4-NX-M0, T4-NX-MX

b Type of planned chemotherapy was defined by metastatic status: palliative, M+; non-palliative, M0, T2 or higher, any N; other

${ }^{c}$ Current or continuing comorbidities. Selected comorbidities presented from non-mutually exclusive groups

Table 2 Chemotherapy regimens received

\begin{tabular}{lccc}
\hline & $\begin{array}{l}\text { Received G-CSF } \\
\text { primary } \\
\text { prophylaxis } \\
(N=70)\end{array}$ & $\begin{array}{l}\text { No G-CSF } \\
\text { primary } \\
\text { prophylaxis } \\
(N=129)\end{array}$ & $\begin{array}{l}\text { Total } \\
(N=199)\end{array}$ \\
\hline $\begin{array}{l}\text { Patients who started } \\
\text { cycle 1 on their } \\
\text { planned regimen }\end{array}$ & $70(100 \%)$ & $128(99 \%)$ & $198(99 \%)$ \\
$n(\%)$ & & & \\
DCF & $38(54 \%)$ & $16(13 \%)$ & $54(27 \%)$ \\
EOX & $1(1 \%)$ & $14(11 \%)$ & $15(8 \%)$ \\
ECF & $1(1 \%)$ & $13(10 \%)$ & $14(7 \%)$ \\
ELF & 0 & $14(11 \%)$ & $14(7 \%)$ \\
FOLFOX & $3(4 \%)$ & $11(9 \%)$ & $14(7 \%)$ \\
CF & 0 & $10(8 \%)$ & $10(5 \%)$ \\
IF & $1(1 \%)$ & $9(7 \%)$ & $10(5 \%)$ \\
ECX & $2(3 \%)$ & $7(5 \%)$ & $9(5 \%)$ \\
LV5FU2 & 0 & $7(5 \%)$ & $7(4 \%)$ \\
XP & 0 & $7(5 \%)$ & $7(4 \%)$ \\
DC & $3(4 \%)$ & 0 & $3(2 \%)$ \\
DX & 0 & $3(2 \%)$ & $3(2 \%)$ \\
FEM & 0 & $3(2 \%)$ & $3(2 \%)$ \\
DF & $1(1 \%)$ & $1(1 \%)$ & $2(1 \%)$ \\
EOF & 0 & $1(1 \%)$ & $1(1 \%)$ \\
FAM & $16(23 \%)$ & $4(3 \%)$ & $20(10 \%)$ \\
Other & $3(4 \%)$ & $8(6 \%)$ & $11(6 \%)$ \\
Single agent & 0 & & \\
\hline
\end{tabular}

Two patients changed from cisplatin to carboplatin during the study: one received ECX and the other received DCF in the first cycle

$D C F$ docetaxel, cisplatin, 5-FU; $E C F$ epirubicin, cisplatin, 5-FU; $E C X$ epirubicin, cisplatin, capecitabine; $C F$ cisplatin, 5-FU; $D F$ docetaxel, 5-FU; $I F$ irinotecan, 5-FU, leucovorin; $E L F$ etoposide, leucovorin, 5-FU; $X P$ capecitabine, cisplatin; FAM 5-FU, doxorubicin, mitomycin $\mathrm{C} ; E O X$ epirubicin, oxaliplatin, capecitabine; FEM 5-FU, epirubicin, mitomycin C; $D X$ docetaxel, capecitabine; $D C$ docetaxel, cisplatin; EOF epirubicin, oxaliplatin, 5-FU; FOLFOX leucovorin, 5-FU, oxaliplatin; LV5FU2 leucovorin, $5-\mathrm{FU}$

${ }^{a}$ Chemotherapy regimen received in cycle $1 ; 181$ patients $(91 \%)$ ended the observational period on their planned chemotherapy regimen a
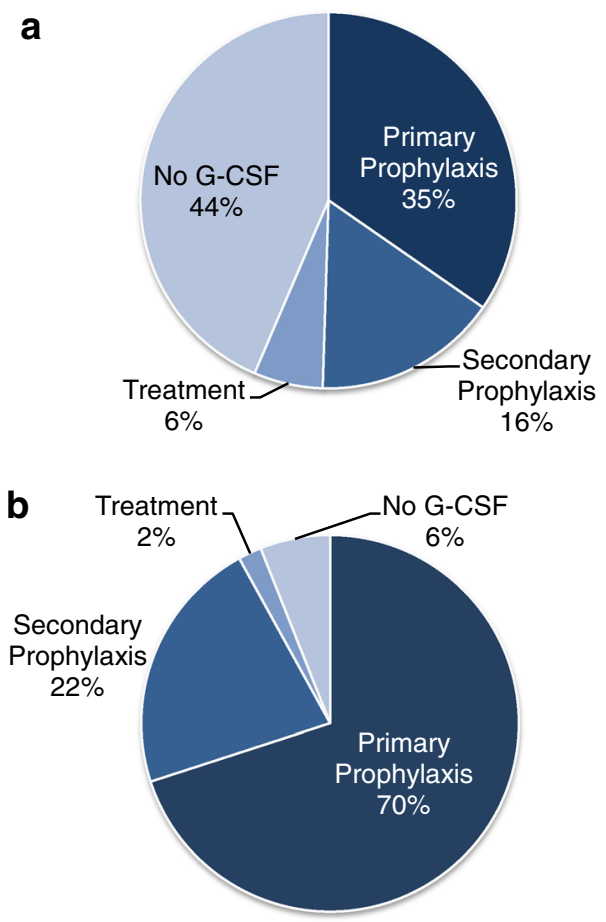

Fig. 2 a G-CSF use in patients with gastric cancer identified as at high risk for febrile neutropenia $(n=199)$. b G-CSF use in patients with gastric cancer identified as at high risk for febrile neutropenia who received DCF $(n=54)$

$0-1,54 \%$ had metastatic disease and $47 \%$ had no prior treatment. Of the $53 \%$ who were previously treated, $60 \%$ had prior surgery and $24 \%$ had received chemotherapy with or without radiotherapy.

Seventy patients $(35 \%)$ received G-CSF primary prophylaxis. At study entry, patients who received G-CSF primary prophylaxis were slightly younger (mean age 60 vs. 64 years) and were more likely to have ECOG scores of 0 or 1 ( 86 vs. $81 \%$ ), cardiovascular comorbidity (78 vs. 
$64 \%$ ), advanced tumor stage (stage T4 30 vs. $18 \%$ ), and prior therapy (59 vs. $50 \%$ ) than patients who did not receive primary prophylaxis (Table 1 ).

\section{Chemotherapy regimens}

Planned chemotherapy was administered with palliative intent (defined by a positive metastatic status) for $54 \%$ of patients and non-palliative intent for $34 \% \quad(n=67)$ (Table 1). A total of 27 different backbone regimens were planned and received (ten triplet, 12 doublet, and five single-drug regimens) (see Table 2 for most common regimens). The most common regimen received was DCF (27\%; $n=54)$, which was therefore selected for additional analyses as described below.

Of those who received DCF, 13 (24\%) received standard DCF [10] and $41(76 \%)$ received modified DCF. There was a large variation among the different modifications to DCF used in the study. Several modifications to DCF have been reported, but for the patients in the study we were able to find only two that exactly matched previously published modified regimens: reduced dose intensity of all three agents given every 3 weeks [20] $(n=2)$, and reduced dose intensity of docetaxel and cisplatin, with leucovorin added, given every 2 weeks [21] $(n=4)$. Other modifications included giving only 1 cycle of standard DCF ( $n=2$ ) or using standard doses of DCF given every 4 weeks $(n=5)$. Overall, it was difficult to exactly match most of the modifications to DCF used in the study to a published modified regimen, with further changes made both to chemotherapy doses and cycle lengths. Ten percent of patients in the study were allocated to the 'Other' group, because the regimen they received could not be matched to a known standard regimen. These were a mixture of doublet and triplet regimens, and half of the patients received an oxaliplatin/irinotecan-based combination.

\section{G-CSF usage and FN}

The analysis of overall G-CSF use was prespecified and was conducted using programmatically derived definitions of primary prophylaxis, secondary prophylaxis, treatment, and no G-CSF as described in the "Methods." Despite all patients having been assessed as at high risk for FN, only $35 \%$ of patients overall were given G-CSF primary prophylaxis (Fig. 2a). Patients who received G-CSF primary prophylaxis were more commonly administered pegfilgrastim $(77 \%, n=54)$ than a daily G-CSF $(23 \%, n=16)$. Primary prophylaxis with daily G-CSF only (i.e., no pegfilgrastim) was administered for a mean (SD) of 5.2 (1.6) days per cycle. Of patients who received the DCF regimen in cycle $1,70 \%$ received G-CSF primary prophylaxis (Fig. 2b).
FN

FN in any cycle occurred in 14 patients overall (7\%). Patients who received G-CSF primary prophylaxis were more likely to have some patient FN risk factors such as cardiovascular comorbidity, docetaxel-based chemotherapy regimens (64 vs. $21 \%$ ), and DCF (54 vs. $13 \%$ ) than those who did not receive G-CSF primary prophylaxis (Table 2). FN occurred in $7 / 70$ patients $(10 \%)$ who received G-CSF primary prophylaxis and $7 / 129$ patients $(5 \%)$ who did not receive G-CSF primary prophylaxis. Most FN events occurred in patients who received DCF (9/14 events, $64 \%)$. All 14 patients with FN had only one episode; for 12 of these patients, hospitalization was the primary management of FN, with a median $(\mathrm{Q} 1, \mathrm{Q} 3)$ stay of $7(6,10)$ days.

In a post hoc analysis, we evaluated on-schedule G-CSF usage in patients who experienced FN. In this analysis, narrower definitions of G-CSF prophylaxis were used (see "Methods"), which were aligned more closely to appropriate product use as described in the product labeling, including initiation within 3 days of the end of chemotherapy and administration of at least three consecutive doses (for daily G-CSF only). Of the 14 patients who experienced FN, most (12 [86 \%]) had not been given on-schedule G-CSF prophylaxis up until the time of their event. Of the nine patients who experienced $\mathrm{FN}$ while receiving the DCF regimen, seven $(78 \%)$ had not been given on-schedule G-CSF primary prophylaxis up until the time of their event. Furthermore, despite having experienced FN, $86 \%$ of the 14 patients were not given on-schedule G-CSF prophylaxis in the subsequent cycle after the FN event.

\section{Safety}

Adverse drug reactions (considered by the investigator to be associated with G-CSF) were reported in three of 78 patients who received pegfilgrastim (two events of bone pain and one event each of back pain, leukocytosis, and chest discomfort). There were no adverse drug reactions reported in the 34 patients who received filgrastim. No serious adverse drug reactions were reported.

\section{Discussion}

In this observational study conducted in patients with gastric cancer who were identified by the investigator as being at high risk for $\mathrm{FN}$, the majority (65\%) did not receive G-CSF primary prophylaxis. EORTC clinical practice guidelines [16] recommend primary prophylaxis with G-CSF in patients identified as being at high risk ( $\geq 20 \%$ risk) for the development of $\mathrm{FN}$ based on their treatment regimen and other individual patient factors. At 
the time this study was conducted, practice guidelines did not specifically identify chemotherapy regimens associated with high risk in patients with gastric cancer; however, in the updated 2010 guidelines [15], regimens including DCF, TCF, and TC were classified as high risk. LV5FU (leucovorin, 5-FU) and ECF (epirubicin, cisplatin, 5-FU) were also classified in the guidelines as high-risk regimens, but evidence from the primary literature indicates they carry an FN risk of 9 and $18 \%$, respectively [22, 23]. DCF was the most common regimen used in the study, and many modifications of DCF were used, including many variations to the published standard or modified schedules. Most other regimens used were triple or double combinations. Of note, 27 different regimens were used, highlighting the highly variable nature of chemotherapy practice in gastric cancer. In general, we would like to emphasize that the variety of chemotherapy regimens used, the frequent modifications to standard chemotherapy regimens, and the presence of patient risk factors contribute to the difficulty in assessing FN risk in gastric cancer.

As expected, patient risk factors for FN were common in the study population, including advanced disease, older age, cardiovascular comorbidity, and prior chemotherapy. Despite all patients in the study meeting the guideline criteria for receipt of primary prophylaxis $(\geq 20 \%$ risk of FN), only $35 \%$ of patients received G-CSF. Patients selected for G-CSF primary prophylaxis were slightly younger, had better performance status, had more cardiovascular comorbidity, were more likely to have been previously receiving chemotherapy, and were more likely to receive a docetaxel-containing chemotherapy regimen. In addition, patients given G-CSF primary prophylaxis were more frequently given pegfilgrastim than filgrastim or lenograstim. Of those given daily G-CSF, the mean length of administration was only 5.2 days, which has previously been established to provide lower protection than one dose of pegfilgrastim [24-27]. Of those who received the DCF regimen, the use of G-CSF primary prophylaxis was higher, though a full $30 \%$ of patients were not administered protection against neutropenia.

FN occurred in $10 \%$ of those who received primary prophylaxis and $5 \%$ of those who did not. We note that FN rates between those who did and did not receive primary prophylaxis should not be compared, as the populations were not similar in this observational study because patients at higher risk were more likely to receive an intervention. A majority of those who had $\mathrm{FN}$ had received the DCF regimen. Of those who experienced FN despite having received primary prophylaxis, we note that the vast majority did not receive G-CSF in accordance with the product labeling (i.e., timing of initiation was too late and/ or an insufficient number of consecutive doses were administered). Use of secondary prophylaxis to protect patients who experienced a prior FN event was also very low in contrast to guideline recommendations. This study sample was large and representative of the European gastric cancer population receiving chemotherapy, including patients enrolled from seven countries; therefore, the results are likely to be relevant to the wider European population.

The limitations of this study include its observational design, in which therapeutic intervention was subject to clinical judgment such that patients at higher risk were more likely to receive an intervention. In addition, investigators could use alternative clinical therapeutic options to prevent FN, including antibiotic prophylaxis, dose reductions, and dose delays. Therefore, the patient populations that did and did not receive primary prophylaxis were not similar (and adjustment for all known and unknown confounding factors was not possible). Another limitation of the study is that the FN prophylaxis decision is often secondary to reimbursement policy in European countries and may not reflect the physician choice or the physician's knowledge of clinical guideline recommendations. Finally, we note that the population studied includes only those patients receiving myelosuppressive chemotherapy, and therefore may not be representative of the entire gastric cancer population.

In conclusion, the results of this study reveal a high use of myelotoxic treatment regimens in gastric cancer in Europe and low adherence to clinical practice guidelines for the use of primary and secondary prophylaxis for FN. Improved risk assessment and appropriate targeting of G-CSF primary prophylaxis to high-risk patients is needed in gastric cancer, particularly among patients receiving DCF.

Acknowledgments This work was supported by Amgen (Europe) $\mathrm{GmbH}$. Wanda J. Krall, PhD on behalf of Amgen (Europe), and James $\mathrm{O}$ 'Kelly, $\mathrm{PhD}$ of Amgen (Europe) supported the writing of this manuscript. In collaboration with the academic investigators, Amgen (the sponsor) designed the study, gathered the data, conducted the statistical analyses, and interpreted the results. All authors participated in the design or execution of the study, contributed to interpretation of the data, approved the final draft of the manuscript, and made the decision to submit it for publication.

Conflict of interest Ewa Kalinka-Warzocha received honoraria from Amgen. Laurent Mineur received travel reimbursement from Amgen. Lucy DeCosta and Florian D. Vogl are employees of Amgen and hold Amgen stock. Javier Gallego Plazas, Tomas Salek, Alain Hendlisz, and Rodolfo Passalacqua have nothing to disclose.

\section{References}

1. GLOBOCAN. http://globocan.iarc.fr/factsheets/cancers/stomach. asp. Accessed 19 June 2013.

2. EUCAN. http://eco.iarc.fr/EUCAN/Cancer.aspx?Cancer=8\#blocktable-a. Accessed 17 April 2013. 
3. Van Cutsem E, Dicato M, Geva R, Arber N, Bang Y, Benson A, et al. The diagnosis and management of gastric cancer: expert discussion and recommendations from the 12th ESMO/World Congress on Gastrointestinal Cancer, Barcelona, 2010. Ann Oncol. 2011;22(Suppl 5):v1-9. doi:10.1093/annonc/mdr284.

4. Okines A, Verheij M, Allum W, Cunningham D, Cervantes A. Gastric cancer: ESMO Clinical Practice Guidelines for diagnosis, treatment and follow-up. Ann Oncol. 2010;21(Suppl 5):v50-4. doi:10.1093/annonc/mdq164.

5. Van Cutsem E, Van de Velde C, Roth A, Lordick F, Kohne CH, Cascinu S, et al. Expert opinion on management of gastric and gastro-oesophageal junction adenocarcinoma on behalf of the European Organisation for Research and Treatment of Cancer (EORTC)-gastrointestinal cancer group. Eur $\mathrm{J}$ Cancer. 2008;44(2):182-94. doi:10.1016/j.ejca.2007.11.001.

6. Wagner AD, Unverzagt S, Grothe W, Kleber G, Grothey A, Haerting $\mathbf{J}$ et al. Chemotherapy for advanced gastric cancer. Cochrane Database Syst Rev. 2010. (3):CD004064. doi:10.1002/ 14651858.CD004064.pub3.

7. Wagner AD, Grothe W, Haerting J, Kleber G, Grothey A, Fleig WE. Chemotherapy in advanced gastric cancer: a systematic review and meta-analysis based on aggregate data. J Clin Oncol. 2006;24(18):2903-9. doi:10.1200/JCO.2005.05.0245.

8. Bang YJ, Van Cutsem E, Feyereislova A, Chung HC, Shen L, Sawaki A, et al. Trastuzumab in combination with chemotherapy versus chemotherapy alone for treatment of HER2-positive advanced gastric or gastro-oesophageal junction cancer (ToGA): a phase 3, open-label, randomised controlled trial. Lancet. 2010;376(9742):687-97. doi:10.1016/S0140-6736(10)61121-X.

9. Al-Batran SE, Hartmann JT, Probst S, Schmalenberg H, Hollerbach $\mathrm{S}$, Hofheinz R, et al. Phase III trial in metastatic gastroesophageal adenocarcinoma with fluorouracil, leucovorin plus either oxaliplatin or cisplatin: a study of the Arbeitsgemeinschaft Internistische Onkologie. J Clin Oncol. 2008;26(9):1435-42. doi:10.1200/JCO.2007.13.9378.

10. Van Cutsem E, Moiseyenko VM, Tjulandin S, Majlis A, Constenla M, Boni C, et al. Phase III study of docetaxel and cisplatin plus fluorouracil compared with cisplatin and fluorouracil as firstline therapy for advanced gastric cancer: a report of the V325 Study Group. J Clin Oncol. 2006;24(31):4991-7. doi:10.1200/ JCO.2006.06.8429.

11. Kuderer NM, Dale DC, Crawford J, Cosler LE, Lyman GH. Mortality, morbidity, and cost associated with febrile neutropenia in adult cancer patients. Cancer. 2006;106(10):2258-66. doi:10. 1002/cncr.21847.

12. Pettengell R, Schwenkglenks M, Leonard R, Bosly A, Paridaens $\mathrm{R}$, Constenla M, et al. Neutropenia occurrence and predictors of reduced chemotherapy delivery: results from the INC-EU prospective observational European neutropenia study. Support Care Cancer. 2008;16(11):1299-309. doi:10.1007/s00520-008-0430-4.

13. Chirivella I, Bermejo B, Insa A, Perez-Fidalgo A, Magro A, Rosello $\mathrm{S}$, et al. Optimal delivery of anthracycline-based chemotherapy in the adjuvant setting improves outcome of breast cancer patients. Breast Cancer Res Treat. 2009;114(3):479-84. doi:10.1007/s10549-008-0018-1.

14. Lyman GH, Dale DC, Culakova E, Poniewierski MS, Wolff DA, Kuderer NM, et al. The impact of the granulocyte colony-stimulating factor on chemotherapy dose intensity and cancer survival: a systematic review and meta-analysis of randomized controlled trials. Ann Oncol. 2013; , doi:10.1093/annonc/mdt226.

15. Aapro MS, Bohlius J, Cameron DA, Dal Lago L, Donnelly JP, Kearney N, et al. 2010 update of EORTC guidelines for the use of granulocyte-colony stimulating factor to reduce the incidence of chemotherapy-induced febrile neutropenia in adult patients with lymphoproliferative disorders and solid tumours. Eur J Cancer. 2011;47(1):8-32. doi:10.1016/j.ejca.2010.10.013.
16. Aapro MS, Cameron DA, Pettengell R, Bohlius J, Crawford J, Ellis $\mathrm{M}$, et al. EORTC guidelines for the use of granulocytecolony stimulating factor to reduce the incidence of chemotherapy-induced febrile neutropenia in adult patients with lymphomas and solid tumours. Eur J Cancer. 2006;42(15):2433-53. doi:10. 1016/j.ejca.2006.05.002.

17. Chan A, Verma S, Loibl S, Crawford J, Choi MR, Dreiling L, et al. Reporting of myelotoxicity associated with emerging regimens for the treatment of selected solid tumors. Crit Rev Oncol Hematol. 2012;81(2):136-50. doi:10.1016/j.critrevonc.2011.03. 003.

18. Amgen. Neulasta (R). Summary of Product Characteristics. 2013. Available from: http://www.medicines.org.uk/emc/medicine/ 11783/SPC/NEULASTA/.

19. Amgen. Neupogen (R). Summary of Product Characteristics (EU). 2014. Available from: http://www.medicines.org.uk/EMC/ medicine/23292/SPC/Neupogen+Singleject+30+MU+ $(0.6+\mathrm{mg}+$ $\mathrm{ml}) /$.

20. Ozdemir NY, Abali H, Oksuzoglu B, Budakoglu B, Uncu D, Guler T, et al. The efficacy and safety of reduced-dose docetaxel, cisplatin, and 5-fluorouracil in the first-line treatment of advanced stage gastric adenocarcinoma. Med Oncol. 2010;27(3):680-4. doi:10.1007/s12032-009-9268-y.

21. Shah M, Shibata S, Stoller R, Kemeny M, Ritch P, Krishnamurthi S et al. Random assignment multicenter phase II study of modified docetaxel, cisplatin, fluorouracil ( $\mathrm{mDCF}$ ) versus DCF with growth factor support (GCSF) in metastatic gastroesophageal adenocarcinoma (GE). J Clin Oncol 28:15s (suppl; abstr 4014). 2010.

22. Roth AD, Fazio N, Stupp R, Falk S, Bernhard J, Saletti P, et al. Docetaxel, cisplatin, and fluorouracil; docetaxel and cisplatin; and epirubicin, cisplatin, and fluorouracil as systemic treatment for advanced gastric carcinoma: a randomized phase II trial of the Swiss Group for Clinical Cancer Research. J Clin Oncol. 2007;25(22):3217-23. doi:10.1200/JCO.2006.08.0135.

23. Bouche O, Raoul JL, Bonnetain F, Giovannini M, Etienne PL, Lledo $\mathrm{G}$, et al. Randomized multicenter phase II trial of a biweekly regimen of fluorouracil and leucovorin (LV5FU2), LV5FU2 plus cisplatin, or LV5FU2 plus irinotecan in patients with previously untreated metastatic gastric cancer: a Federation Francophone de Cancerologie Digestive Group Study-FFCD 9803. J Clin Oncol. 2004;22(21):4319-28. doi:10.1200/JCO. 2004.01.140.

24. Morrison VA, Wong M, Hershman D, Campos LT, Ding B, Malin J. Observational study of the prevalence of febrile neutropenia in patients who received filgrastim or pegfilgrastim associated with 3-4 week chemotherapy regimens in community oncology practices. J Manag Care Pharm. 2007;13(4):337-48.

25. von Minckwitz G, Schwenkglenks M, Skacel T, Lyman GH, Pousa AL, Bacon P, et al. Febrile neutropenia and related complications in breast cancer patients receiving pegfilgrastim primary prophylaxis versus current practice neutropaenia management: results from an integrated analysis. Eur J Cancer. 2009;45(4):608-17. doi:10.1016/j.ejca.2008.11.021.

26. Weycker D, Hackett J, Edelsberg JS, Oster G, Glass AG. Are shorter courses of filgrastim prophylaxis associated with increased risk of hospitalization? Ann Pharmacother. 2006;40(3):402-7. doi:10.1345/aph.1G516.

27. Almenar D, Mayans J, Juan O, Bueno JM, Lopez JI, Frau A, et al. Pegfilgrastim and daily granulocyte colony-stimulating factor: patterns of use and neutropenia-related outcomes in cancer patients in Spain-results of the LEARN Study. Eur J Cancer Care (Engl). 2009;18(3):280-6. doi:10.1111/j.1365-2354.2008. 00959.x. 